\title{
Philosophiques
}

\section{Deux conceptions de l'interprétation des récits de fiction}

\section{Jérôme Pelletier}

Volume 32, numéro 1, printemps 2005

Questions d'interprétation

URI : https://id.erudit.org/iderudit/011062ar

DOI : https://doi.org/10.7202/011062ar

Aller au sommaire du numéro

Éditeur(s)

Société de philosophie du Québec

ISSN

0316-2923 (imprimé)

1492-1391 (numérique)

Découvrir la revue

Citer cet article

Pelletier, J. (2005). Deux conceptions de l'interprétation des récits de fiction. Philosophiques, 32(1), 39-54. https://doi.org/10.7202/011062ar

\section{Résumé de l'article}

Deux conceptions de l'interprétation des récits de fiction sont présentées : la conception " communicationnelle " de Currie et la conception " émotionnelle " de Velleman. Selon Currie, interpréter un récit de fiction requiert de rapporter les événements du récit aux intentions narratives de l'auteur (inféré). Cette conception suppose que l'interprète se représente le contexte pragmatique de production du récit et va à l'encontre d'un certain nombre de recherches empiriques montrant que le lecteur d'un récit de fiction ne construit que très rarement une représentation du contexte pragmatique de sa production. Selon Velleman, l'interprétation requiert de la part du lecteur ou du spectateur qu'il reconnaisse dans la séquence émotionnelle qu'il éprouve à la lecture ou au spectacle d'une fiction une séquence émotionnelle familière. Selon cette conception, les événements narrés sont interprétés en fonction de la manière dont ils « résonnent » émotionnellement dans l'esprit du lecteur ou du spectateur. 


\title{
Deux conceptions de l'interprétation des récits de fiction
}

\author{
JÉRÔME PELLETIER \\ Université de Brest et Institut Jean-Nicod (Paris) \\ Jerome.Pelletier@ehess.fr
}

\begin{abstract}
RÉSUMÉ. - Deux conceptions de l'interprétation des récits de fiction sont présentées : la conception «communicationnelle » de Currie et la conception «émotionnelle » de Velleman. Selon Currie, interpréter un récit de fiction requiert de rapporter les événements du récit aux intentions narratives de l'auteur (inféré). Cette conception suppose que l'interprète se représente le contexte pragmatique de production du récit et va à l'encontre d'un certain nombre de recherches empiriques montrant que le lecteur d'un récit de fiction ne construit que très rarement une représentation du contexte pragmatique de sa production. Selon Velleman, l'interprétation requiert de la part du lecteur ou du spectateur qu'il reconnaisse dans la séquence émotionnelle qu'il éprouve à la lecture ou au spectacle d'une fiction une séquence émotionnelle familière. Selon cette conception, les événements narrés sont interprétés en fonction de la manière dont ils « résonnent » émotionnellement dans l'esprit du lecteur ou du spectateur.
\end{abstract}

\begin{abstract}
I discuss two ways one may explain how we interpret the content of a fictional. In the first, the interpreter's task aims at deciding what is true in a fictional story by figuring out the narrative intentions behind its production. Narrative interpretation is a matter of figuring out the story-telling intentions of the (implied) author of the work. This is Currie's intentionalist model of narrative interpretation, a conception I present and discuss on the basis of experimental results in the psychology of language. In the second, narrative interpretation is explained by reference to the emotions of the reader or the viewer of the fictional narrative. It is on the basis of his emotional sensibility, of how things feel to him that the reader or the viewer is led to understand the narrated events. This is Velleman's emotional theory of narrative understanding. Finally, I compare both conceptions.
\end{abstract}

\section{Introduction}

L'information communiquée par un récit, qu'il soit littéraire ou cinématographique, ne se limite pas à une liste d'événements : un récit raconte le déroulement de ces événements, leur ordre de succession, leur organisation les uns par rapport aux autres. L'acte de raconter vise ainsi à communiquer plus que ce que les mots ou séquences d'images figurant dans un récit signifient lorsque ceux-ci sont considérés indépendamment les uns des autres. Ce supplément communiqué est, le plus souvent, ce qui permet d'expliquer les événements narrés. De manière générale, ce supplément représente pour le lecteur ou le spectateur un supplément d'intelligibilité et de signification des événements narrés. Appelons "histoire» ce supplément communiqué par un récit : l'histoire peut être l'intrigue, la trame causale des événements, la logique des relations entre les événements narrés, mais aussi, plus généralement, le sens des 
événements racontés. Et appelons «interprétation" l'ensemble des processus qui, à partir d'un récit, rendent les événements narrés intelligibles et aboutissent à une histoire.

Les lecteurs d'un récit de fiction ou les spectateurs d'un film de fiction sont naturellement conduits à dériver un ensemble de jugements sur ce qui est fictionnellement vrai dans le livre ou dans le film ${ }^{1}$. Que sont ces "vérités de fiction" ? Considérons le conte de Perrault sur Cendrillon. Ce conte contient de nombreuses faussetés. Cendrillon n'existe pas et les citrouilles ne se transforment pas en carrosses. Mais ces faussetés réelles sont vraies dans le conte de Perrault : il est vrai dans ce conte que Cendrillon existe et que les citrouilles peuvent se transformer en carrosses. Toutes les vérités de fiction ne sont pas si évidentes. En particulier, nombreux sont les événements narrés dans les fictions dont la logique n'est pas donnée de manière explicite par l'auteur. Un romancier peut très bien présenter une succession de scènes sans lien causal évident, un cinéaste peut, par le montage, juxtaposer deux scènes sans lien causal apparent. Comment expliquer que le lecteur ou le spectateur pourra être conduit à établir une connexion entre ces événements ?

Dans ce qui suit, je présente deux réponses possibles à cette question ainsi que les conceptions particulières de l'interprétation des récits de fiction qui sous-tendent ces réponses. Je présente tout d'abord la conception «communicationnelle» de l'interprétation de Gregory Currie. Selon cette conception, l'interprétation requiert de la part du lecteur ou du spectateur qu'il lise le texte ou qu'il voie le film comme le résultat d'intentions communicatives particulières, comme le résultat de l'intention de la part de l'auteur du texte ou du film de communiquer au moyen de ce texte ou ce film une histoire spécifique. Je présente ensuite une critique de la conception communicationnelle et une explication alternative de la manière dont un récit de fiction peut avoir un sens pour un lecteur ou un spectateur : la conception «émotionnelle» de l'interprétation des récits de fiction.

\section{La conception communicationnelle de l'interprétation}

\section{La représentation du contexte pragmatique}

Selon Currie et Jureidini (2003), on ne peut expliquer le fait que le lecteur ou le spectateur établisse des connexions entre des événements apparemment disjoints de manière causale sans admettre que ce lecteur ou ce spectateur se représente, peut-être de manière tacite, le texte ou le film comme une narration, c'est-à-dire comme la production d'un agent ayant des intentions narratives. Selon cette conception, le lecteur ou le spectateur construirait une représentation, peut-être non consciente, du contexte pragmatique dans lequel le texte ou le film

1. Le travail interprétatif commence véritablement lorsqu'il s'agit pour le lecteur ou le spectateur de saisir un ensemble de "vérités de fiction ». C'est à cette dimension du travail interprétatif du lecteur ou du spectateur que je m'intéresse dans cet article. Je laisse particulièrement de côté le travail interprétatif nécessaire à la détermination des propriétés esthétiques ou stylistiques d'une fiction littéraire ou filmique. 
est enchâssé. Par exemple, dans le cas d'une œuvre littéraire, le lecteur construirait la représentation d'un auteur - ce que Currie appelle à la suite de Booth (1961) l'auteur "inféré» ${ }^{2}$ - qui produit le texte pour un lecteur.

Currie et Jureidini prennent l'exemple suivant : dans The Stalls of Barchester Cathedral, M. R. James révèle au lecteur qu'un archevêque âgé est mort au cours d'une chute et que le $\mathrm{D}^{\mathrm{r}}$ Hayes, qui attendait depuis de nombreuses années ce moment, lui succéda. Le lecteur forme alors la conviction que le $\mathrm{D}^{\mathrm{r}}$ Hayes est responsable de la mort de l'archevêque. Selon Currie et Jureidini, en juxtaposant dans le même paragraphe la nouvelle de la mort de l'archevêque et la réaction du $\mathrm{D}^{\mathrm{r}}$ Hayes à cette nouvelle « debout silencieux devant la fenêtre ", James indique au lecteur une connexion entre les deux événements, pour laquelle il n'offre d'autre preuve que sa propre intention que le lecteur voie les choses ainsi.

La situation, selon Currie et Jureidini, est encore plus flagrante dans La Dame de Shangaï d'Orson Wells. Dans ce film, la juxtaposition de la scène où un personnage féminin appuie sur un bouton et de la scène où une voiture a un accident suggère fortement au spectateur d'établir une connexion entre les deux événements bien que les deux scènes se déroulent à distance l'une de l'autre. La raison de l'inférence du spectateur ne se trouve pas dans les événements eux-mêmes mais, selon Currie et Jureidini, dans le fait que le spectateur reconnaît derrière la juxtaposition de ces deux événements l'intention de l'auteur (inféré) du film. Le spectateur infère l'existence d'un lien entre les deux événements parce qu'il reconnaît, d'une part, que le monde de la fiction est gouverné par l'intention d'une intelligence organisatrice et, d'autre part, que l'auteur (inféré) de la fiction a l'intention de raconter une histoire dans laquelle ces deux événements seraient connectés.

Dans une fiction, rien ne peut constituer un indice de l'histoire à moins que le lecteur ou le spectateur ne reconnaisse que l'auteur du récit a placé cet indice avec l'intention qu'il soit traité comme tel par le lecteur ou le spectateur. Dans la conception de Currie et Jureidini, c'est dans la mesure où une information donnée dans le récit - un événement, ce que dit un personnage, une juxtaposition de scènes - est rapportée aux intentions narratives de son auteur (inféré) que cette information peut jouer, pour le lecteur ou le spectateur, le rôle d'indice de l'histoire.

\section{Interprétation d'une œuvre et interprétation du comportement}

Pour Currie (1995a), l'interprétation requiert donc de faire référence aux intentions narratives de l'auteur (inféré). Interpréter un récit revient à formuler des

2. L' «auteur inféré» d'une œuvre ne correspond pas nécessairement à son auteur réel, et encore moins lorsque figure dans l'œuvre un narrateur explicite, à ce narrateur. Pour Currie, l'auteur inféré est l'agent responsable du récit en tant que celui-ci est un récit de fiction alors que son éventuel narrateur explicite est censé raconter ce qui est de la fiction comme s'il en avait une connaissance réelle. (Cf. par exemple, Currie [1995b, p. 20]). Pour la défense du concept d'«auteur inféré» d'un film, voir Currie (1995a, p. 243-280). 
hypothèses sur les intentions de cet auteur (inféré). Et le contenu des intentions de l'auteur (inféré) est le contenu de l'histoire. Cette démarche conduit Currie à voir dans l'interprétation d'une œuvre et l'interprétation du comportement deux projets structurellement similaires. De la même manière que l'interprétation du comportement suppose - au moins dans une certaine conception de formuler des hypothèses sur les états mentaux qui ont causé ce comportement, l'interprétation d'une œuvre suppose de formuler des hypothèses sur les causes intentionnelles de l'œuvre.

À partir de l'observation qu'un texte ou un film est le résultat d'un certain comportement, et à partir de l'analogie entre l'interprétation du comportement et l'interprétation d'une œuvre, Currie défend la thèse que l'interprétation des récits est une forme d'explication. Interpréter un récit revient à expliquer le comportement dont il résulte. Une interprétation est une tentative pour expliquer un récit en attribuant à l'auteur (inféré) des intentions narratives particulières de même que l'attribution à l'agent de croyances et de désirs est une tentative pour expliquer un comportement ${ }^{3}$.

La visée explicative de l'interprétation soumet l'interprétation d'un récit aux mêmes contraintes que toute autre forme d'explication. La recherche de simplicité, de plausibilité et d'une ampleur explicative gouvernent l'interprétation. Ainsi, Currie note que si rien, dans le texte de Doyle, n'exclut d'interpréter les histoires de Sherlock Holmes comme les divagations de Watson, une telle interprétation, certes compatible avec l'ensemble du texte, ne serait pas explicative de ce texte. Cette interprétation doit être rejetée en particulier parce qu'elle ne donne pas un sens plausible et simple au texte ${ }^{4}$.

\section{Interprétation et simulation}

Selon Currie, interpréter un texte ou un film revient à formuler des hypothèses sur les causes intentionnelles, sur les intentions narratives de l'auteur (inféré) qui expliquent l'œuvre à interpréter. Pour expliquer le texte ou le film, il faut donc être en mesure d'attribuer des états mentaux — les intentions narratives - à l'auteur (inféré) du texte ou du film.

Il y a depuis quelques années un débat en philosophie de l'esprit sur la manière dont on attribue des états mentaux à autrui, sur la manière dont on découvre ce que les autres pensent ou ressentent ${ }^{5}$. Pour les «théoriciens de la théorie ", nous comprenons les états mentaux d'autrui en appliquant, de manière tacite, une théorie psychologique. Pour les «théoriciens de la simulation ", l'attribution d'états mentaux à une personne se fait par la simulation d'états psychologiques. Sur la base des pensées, émotions ou sentiments que l'on éprouve soi-même au cours de la simulation, on peut comprendre les états mentaux d'autrui.

3. Cf. Currie (1995a, p. 235-242).

4. Cf. Currie (1995a, p. 237).

5. Voir, en particulier, Davies et Stone (1995a, 1995b), Carruthers et Smith (1996) et Dokic et Proust (2002). 
En tant que théoricien de la simulation, Currie voit naturellement dans la simulation la procédure cognitive de connaissance des intentions narratives de l'auteur (inféré) :

Je me place dans la position de l'auteur, pensant à moi-même comme la personne ayant produit le texte et je me demande "Quelles intentions narratives auraient pu me conduire à écrire ce texte? » Si je peux faire tourner une simulation dans laquelle le fait d'avoir ces intentions narratives conduit à la décision ( «off-line») d'écrire ce texte, alors je peux conclure qu'il est au moins plausible que l'auteur a eu ces intentions ${ }^{6}$ (Currie, 1995a, p. 242).

En résumé, la conception communicationnelle de l'interprétation est à la fois une forme d'intentionnalisme interprétatif, une forme d'inférentialisme interprétatif et une forme de simulationnisme interprétatif. La conception communicationnelle est une conception intentionnaliste de l'interprétation, car elle soutient que l'interprétation d'un récit de fiction requiert d'identifier les intentions de communication de son créateur. C'est également une conception inférentielle de l'interprétation puisqu'il s'agit, selon la conception communicationnelle, d'inférer à partir du texte (ou des images) dont il dispose les intentions de son créateur. C'est une conception simulationniste de l'interprétation puisque c'est par le biais de simulations que l'on infère les intentions de l'auteur. Enfin, l'interprétation est conçue comme visant une explication du texte ou du film.

\section{Critique de la conception communicationnelle de l'interprétation}

Si l'on accepte de se limiter aux récits linguistiques ${ }^{7}$, un certain nombre de recherches menées en psychologie du langage ne plaident pas en faveur de la conception communicationnelle de l'interprétation, en particulier en faveur de la thèse que le lecteur construirait une représentation du contexte pragmatique

6. Pour Currie, les fictions encouragent, en plus de la simulation d'être dans la position de l'auteur (inféré), deux autres types de simulation : la simulation d'être dans la position de quelqu'un qui apprend des faits d'une source fiable et la simulation des états mentaux des personnages. Ces trois exercices de la simulation suscités par les œuvres de fiction relèvent de ce que Currie appelle l'imagination "personnelle "; ce sont des exercices de l'imagination dans lesquels le sujet imaginant s'implique, en quelque sorte, de manière personnelle : il s'imagine lui-même être l'auteur (inféré), il s'imagine lui-même être quelqu'un qui a une relation épistémique fiable avec la source du récit, ou il s'imagine lui-même dans la situation de tel ou tel personnage. En revanche, Currie insiste beaucoup sur le fait que, lorsque le lecteur ou le spectateur imagine le contenu fictionnel de l'histoire, il imagine alors simplement que ceci et cela ont lieu sans imaginer avoir un quelconque contact épistémique avec ce qui a lieu. L'imagination du contenu fictionnel de l'histoire est, pour Currie, un exercice de l'imagination "non personnelle". Sur la distinction de l'imagination «personnelle» et «non personnelle», voir Currie (1995a, p. 164-197).

7. Lorsque Currie cherche à construire une théorie pour résoudre le problème de l'interprétation (dans Currie, [1995a, p. 225-259]), il se concentre sur le cas des récits littéraires pour, dans un second temps, appliquer la théorie au cas des récits filmiques. Je ne prétends pas que la critique menée dans cette section de l'adéquation de la théorie de Currie aux récits littéraires constitue également une critique de l'adéquation de sa théorie aux récits filmiques. 
de production du récit. Ces recherches empiriques montreraient plutôt que le lecteur adopte une position imaginaire dans le monde décrit par le récit, une position qui rendrait inutile la représentation d'un auteur (inféré) de l'histoire, dont les intentions seraient de raconter une histoire à un lecteur. Selon ces recherches, à moins que le récit ne mentionne un narrateur de manière explicite, il semble que les lecteurs se placent par l'imagination dans la situation décrite. Dans la terminologie de Duchan et al. (1995), le lecteur d'un récit se placerait lui-même dans une position imaginaire appelée «centre déictique " sur un "plan déictique " par un «déplacement déictique ${ }^{8}$. Selon la théorie du déplacement déictique, le langage permet au lecteur d'avoir une expérience imaginaire des événements ou situations décrites, et cela sans aucun intermédiaire du type "auteur (inféré) ».

Alors que la conception communicationnelle de l'interprétation n'a pas, à ma connaissance, de soutien empirique, la théorie du déplacement déictique semble corroborée par un certain nombre de résultats en psychologie cognitive. Ainsi, Glenberg et al. (1987), ont testé la vitesse de reconnaissance, par des lecteurs, de divers mots figurant dans un récit. À l'issue de ces tests, il apparaît que les lecteurs reconnaissent plus lentement les mots dénotant des objets situés à distance des protagonistes du récit que les mots qui dénotent des objets proches de ceux-ci. De manière générale, les mots qui dénotent des objets présents dans la situation décrite sont reconnus plus rapidement que les mots qui ne dénotent pas des objets présents dans la situation décrite ${ }^{9}$. De même, Zwaan et al. (2000), présentèrent à des sujets des paires de phrases telles que "Tom était en train de jouer du piano. Quand sa mère entra, il arrêta/continua/reprit. » Dans la version avec "arrêta ", l'activité de Tom est discontinue alors que, dans la version avec "continua ", elle perdure malgré l'intervention de l'entrée de la mère. Dans la version avec « reprit », l'action de Tom, après s'être interrompue momentanément, continue. Zwaan et al. testèrent le temps de réponse dans une tâche de reconnaissance des verbes d'action «arrêter/continuer/reprendre» dans les trois versions de la phrase. Les résultats montrèrent que les temps de réponse étaient plus courts dans la version avec «continua » que dans la version avec «arrêta ». Ils furent, en outre, plus courts dans la version avec « reprit» que dans la version avec «arrêta". Zwaan et al. conclurent que la présence d'une action dans une situation, tout comme la réitération de sa présence, facilitent la reconnaissance des verbes indiquant la présence ou la reprise de l'action. Des événements qui perdurent

8. Les termes "déictiques" sont les expressions telles que «je", «tu», «ceci », "cela ", « ici », "maintenant », " aujourd'hui », " hier »... et la détermination de leur référence dépend de la connaissance du contexte de leur énonciation. Alors que dans l'usage ordinaire, la référence des termes déictiques est déterminée à partir d'une connaissance de la perspective spatio-temporelle de leurs énonciateurs ou auditeurs, dans un récit de fiction, selon la théorie du «déplacement déictique ", les lecteurs ou spectateurs déterminent la référence de ces termes en se situant par l'imagination à l'intérieur du monde décrit par le récit.

9. Voir également Morrow et al. (1987), Morrow et al. (1989). 
dans une situation sont plus facilement accessibles au lecteur que des événements qui n'ont plus lieu. Ces résultats empiriques, selon Zwaan et al., étayent la thèse que les lecteurs comprenant un récit se placent eux-mêmes «dans » la situation décrite par le récit ${ }^{10}$.

À la suite de Hamburger (1973), Kuroda (1976) et Banfield (1982), les tenants de la théorie du déplacement déictique soutiennent que lorsque la présence d'un narrateur n'est pas explicitement indiquée dans le texte, la construction de modèles de situations durant la compréhension du texte ${ }^{11}$ doit être conçue analogiquement avec l'expérience d'événements dans le monde réel, sans qu'il soit nécessaire que le contexte pragmatique de communication du récit soit représenté. Selon les théoriciens du déplacement déictique, le récit a précisément pour fonction de rendre possible le déplacement déictique, de guider le lecteur à une place particulière à l'intérieur du monde de l'histoire ${ }^{12}$.

Certes, la théorie du déplacement déictique n'est naturellement pas sans faiblesses ni objections ${ }^{13}$. De plus, l'interprétation à donner des résultats expérimentaux présentés ci-dessus est elle-même l'objet d'une discussion ${ }^{14}$. Il demeure qu'en l'état actuel des connaissances, la théorie du déplacement déictique a un degré de corroboration supérieur à la théorie de la représentation du contexte pragmatique des récits. Comme la théorie de la représentation du contexte pragmatique des récits est une partie essentielle de la conception communicationnelle de l'interprétation, ce constat ne peut que fragiliser cette conception de l'interprétation des récits ${ }^{15}$.

Il reste que l'intuition de Currie sur la similarité structurale entre l'interprétation d'une œuvre et l'interprétation du comportement mérite sans aucun doute d'être approfondie. C'est l'objet de la section suivante que de

10. Voir également Rall et Harris (2000).

11. Suivant la conception dominante en psychologie cognitive, la compréhension linguistique d'un récit suppose la construction de modèles cohérents (ou de représentations mentales) des états de choses décrits dans ce récit. Ce que les psychologues cognitifs appellent un «modèle de la situation » est une représentation mentale des personnes, événements et actions décrits dans le récit.

12. Cf. Segal (1995, p. 67).

13. Il reste en particulier à la théorie du déplacement déictique à préciser ce qu'elle entend par l'adoption, par le lecteur, d'une position imaginaire dans le monde décrit par le récit.

14. Currie (1998b, p. 277) a fait remarquer qu'un défaut majeur de la théorie du déplacement déictique était, dans certains cas, de conduire à admettre des incohérences narratives fondamentales. Currie a également proposé une autre interprétation des résultats expérimentaux présentés plus haut (dans Currie, 1998a, p. 194), une interprétation qu'il juge compatible avec la conception communicationnelle de l'interprétation.

15. Il faudrait également se demander dans quelle mesure la conception communicationnelle de l'interprétation ne suppose pas d'adopter une version particulière de la théorie du déplacement déictique puisque, comme on l'a vu à la note 6 , Currie reconnaît le rôle des exercices des formes «personnelles» de l'imagination dans la détermination du contenu fictionnel de l'histoire. Or les théoriciens du déplacement déictique ne disent autre chose que la compréhension des récits de fiction supposent la mise en jeu de formes "personnelles" de l'imagination. 
développer cette intuition. On verra alors qu'un même point de départ peut conduire à une conception très différente de l'interprétation.

\section{La conception émotionnelle de l'interprétation}

Pour les théoriciens de la simulation, l'attribution d'états psychologiques à d'autres penseurs passe par l'exploitation de nos propres réponses psychologiques. Sans vouloir trancher dans le débat entre théoriciens de la simulation et théoriciens de la théorie, il paraît incontestable, comme le suggèrent les théoriciens de la simulation, que nos états psychologiques, en particulier nos réponses émotionnelles, jouent un rôle dans nos jugements sur les états mentaux d'autrui. Ce sur quoi je souhaite insister dans ce qui suit concerne le rôle joué par nos réponses émotionnelles dans l'attribution d'une intelligibilité aux récits de fiction. C'est un rôle dont l'importance est injustement réduite dans la conception communicationnelle de l'interprétation.

Le rôle des états émotionnels dans nos jugements

sur les états mentaux d'autrui

Nos états émotionnels, s'ils résultent de simulation mentale, peuvent constituer ce à partir de quoi nous attribuons à autrui tel état émotionnel, telle pensée, telle action. Si je souhaite prédire ce qu'une personne va ressentir ou faire en présence d'un cambrioleur, je vais m'imaginer, selon les théoriciens de la simulation, en présence d'un cambrioleur. Si j'éprouve alors un sentiment de panique, je vais attribuer cet état de panique à la personne dont je souhaite prédire les états mentaux. Si je me trouve en train de décider (en imagination) d'appeler la police et d'appeler (en imagination) la police, je vais attribuer cette décision ou cette action à cette personne.

Une des caractéristiques de la simulation mentale est que les réponses psychologiques du simulateur servent de modèles pour inférer ou prédire les états mentaux d'autrui. Le sentiment de panique que j'éprouve, la décision ou l'action d'appeler la police que je me trouve, en imagination, être en train de prendre ou d'accomplir sont considérés par moi-même comme des modèles fiables des états mentaux que je vais attribuer à autrui. Je suppose que les états mentaux d'autrui sont analogues à mes états mentaux, réels ou imaginés, et je projette ces derniers "sur autrui».

Currie a justement insisté sur l'importance de la simulation mentale dans nos interactions avec les fictions. De manière typique, le lecteur ou le spectateur répond aux fictions en s'engageant non pas dans des croyances, mais dans des simulations "off-line». Currie admet en particulier que le lecteur ou le spectateur peut être conduit à simuler la situation de tel ou tel personnage de la manière dont nous simulons les situations d'autrui pour pouvoir leur attribuer des états mentaux ${ }^{16}$. Mais nous disposons de procédures beaucoup plus directes et primitives que la simulation pour attribuer des états

16. Cf. note 6 ci-dessus. 
mentaux à autrui. Plutôt que d'inférer qu'une personne a telle pensée ou tel sentiment par une simulation mentale, mes réponses émotionnelles à l'égard de cette personne, ce que je ressens à son égard, peuvent parfois me permet de lui attribuer directement, sans la médiation d'une simulation, telle pensée ou tel sentiment. Donnons deux exemples : lorsque ma nervosité me fait comprendre que la personne en face de moi est nerveuse, ou lorsque mon sentiment d'être sur la défensive me permet d'attribuer à autrui de l'agressivité à mon égard, mes réponses émotionnelles réelles, hors de toute simulation, me permettent de comprendre certains états mentaux d'autrui. Le premier exemple est un cas de «contagion » émotionnelle et le second un cas de "résonance » émotionnelle. Les processus de "contagion» émotionnelle supposent une identité des émotions ressenties et attribuées tandis que les processus de «résonance » émotionnelle ne supposent pas une telle identité. Citons deux autres exemples de Walton (1999) où des phénomènes de "résonance » émotionnelle constituent la base de mes jugements sur les états mentaux d'une autre personne : c'est parce que je me sens irrité par une personne que je reconnais cette personne comme arrogante, c'est parce que je me sens naturellement porté à venir en aide à une personne que je comprends que cette personne est dans l'abandon. Dans tous ces exemples, la réponse à l'état émotionnel d'autrui précède l'identification de son état émotionnel : nous utilisons nos réponses émotionnelles, nos affects, nos sentiments pour identifier l'état mental d'autrui, nous le comprenons de manière quasi viscérale, à partir de nos affects, de notre sensibilité émotionnelle.

\section{L'interprétation subjective des récits}

De même qu'il y a dans notre sensibilité émotionnelle et dans notre capacité à « résonner » émotionnellement la source d'au moins une partie de notre compréhension d'autrui, il y a dans les émotions que nous éprouvons à la lecture ou au spectacle des récits de fiction la source d'au moins une partie de leur intelligibilité.

L'une des caractéristiques des récits de fiction est qu'ils réussissent à rendre intelligibles à leurs lecteurs ou spectateurs des événements ou des connexions entre événements qui sont soit très peu plausibles, soit difficiles ou impossibles à croire. Faut-il en conclure, avec Currie et Jureidini, que le lecteur ou le spectateur établit des connexions entre des événements apparemment disjoints parce qu'il reconnaît que le monde de la fiction, à la différence du monde réel, est gouverné par une intelligence organisatrice qui a des intentions narratives spécifiques?

Une autre explication semble également pertinente : la connexion entre des événements apparemment disjoints et leur intelligibilité résulteraient non pas d'un lien causal que le lecteur ou le spectateur aurait inféré à partir de la reconnaissance que telle est l'intention de l'auteur (inféré) de l'œuvre, mais parce que ces événements « résonneraient» émotionnellement dans l'esprit du lecteur ou du spectateur selon une séquence émotionnelle classique ou familière. 
L'idée que les émotions sont structurées en séquences a été récemment étayée par DeLancey (2002). À partir de résultats expérimentaux, DeLancey défend la thèse que ce que nous appelons communément «émotion » recouvre en fait un ensemble de choses : des réponses physiologiques (par exemple, des réponses corporelles comme une accélération du cœur), des actions ou comportements stéréotypés associés à ces réponses physiologiques (par exemple, des expressions du visage), et aussi parfois des comportements relationnels (par exemple, la fuite) et des rôles cognitifs particuliers (par exemple, une conscience aiguë du danger). Selon DeLancey, les émotions sont des réponses complexes structurées ou des "programmes d'affect ", c'est-à-dire des ensembles de réponses coordonnées qui, pour la plus grande part, ont une base biologique. Selon DeLancey, il existerait un mécanisme central à la base de ces réponses complexes, un mécanisme qui stockerait les types particuliers de réponses et dirigerait leur déroulement. DeLancey montre également que chaque émotion ou séquence émotionnelle, à la différence d'autres attitudes motivationnelles, est déclenchée par des conditions caractéristiques particulières : la peur, par exemple, est déclenchée dans des conditions caractéristiques.

Si les récits de fiction sont une source majeure de "résonance» émotionnelle pour leur public, à la différence des exemples de "résonance » émotionnelle donnés dans la section précédente, les réponses émotionnelles provoquées par les récits ont une structure complexe. Lire ou voir une fiction ne provoque pas seulement des émotions, mais, à chaque fois, la naissance, l'apogée et la disparition d'une émotion donnée, ainsi que le remplacement de cette émotion par d'autres émotions. À la succession des événements narrés correspond ainsi une succession presque continue d'émotions, chaque émotion suivant le cycle qui lui est propre. À partir de ce constat, Velleman (2002) esquisse une conception émotionnelle de la structure des récits de fiction. La structure d'un récit, ce qu'on appelle aussi l'intrigue, est, selon Velleman, directement connectée aux émotions de son lecteur ou spectateur : le début, le milieu et la fin d'un récit doivent être définis, de manière ultime, dans les termes du déclenchement d'une émotion et de sa résolution. La célèbre thèse d'Aristote, dans sa Poétique, que le drame doit susciter la crainte et la pitié pour ensuite conduire ces deux émotions à une catharsis appropriée, est elle-même fondée, pour Velleman, dans une conception de la nature diachronique des émotions, c'est-à-dire dans la thèse que le début d'une histoire correspond aux circonstances qui déclenchent une émotion ou une séquence d'émotions (crainte et pitié), et dans la thèse que la fin d'une histoire correspond au moment où cette séquence émotionnelle arrive à son terme ${ }^{17}$ (catharsis). L'existence de récits comportant des retours en arrière ou flashbacks ne constitue nullement une difficulté pour la conception émotionnelle de la structure des récits puisque la seule contrainte qui pèse sur la structure du récit est de conduire à une

17. Il faut noter qu'une grande partie de la littérature contemporaine échappe à cette structure classique où la fin de l'histoire correspond à un retour à un équilibre initial. 
résolution de l'émotion ou séquence émotionnelle qu'il a initiée, non de suivre un ordre temporel linéaire ${ }^{18}$.

Dans la mesure où l'émotion ou la succession d'émotions que le lecteur ou le spectateur ressent au récit des événements correspond à une séquence émotionnelle, ou à une succession d'émotions familières à ce lecteur ou spectateur, on peut supposer que la familiarité émotionnelle qu'il ressent constituerait alors la base sur laquelle ce lecteur ou ce spectateur attribuerait du sens aux événements narrés, et cela, indépendamment de l'existence ou de l'inexistence d'une connexion objective de type causal entre ces événements pour ce même lecteur ou spectateur. Selon cette conception émotionnelle de l'interprétation, le lecteur ou le spectateur établirait de manière subjective un lien entre des événements apparemment disjoints, de manière subjective, sur la base des émotions qu'il éprouverait : c'est parce que ses émotions formeraient dans son esprit une séquence familière, c'est parce que les événements narrés auraient une signification émotionnelle pour ce lecteur ou ce spectateur que ces événements, malgré l'apparente absence de liens causaux pour ce même lecteur ou spectateur, seraient reliés entre eux.

De plus, lorsque la succession des événements narrés échappe à la logique et à la rationalité, on peut faire l'hypothèse que les lecteurs ou spectateurs de fiction ne cherchent pas tant une explication causale qu'à établir des liens de signification entre ces événements sur la base des émotions ou séquences émotionnelles ressenties. Si l'occurrence de deux événements sans liens causaux entre eux prend un sens pour le lecteur ou le spectateur, on peut faire l'hypothèse que c'est parce qu'elle suscite dans l'esprit du lecteur ou du spectateur une séquence émotionnelle type, non parce que ce lecteur ou ce spectateur que l'intention de l'auteur (inféré) de l'œuvre était que l'on perçoive un lien de causalité entre ces deux événements. Velleman prend l'exemple de la tragédie d'CEdipe en faisant remarquer que l'absence totale de plausibilité des événements arrivant à CEdipe, en particulier l'ensemble extraordinaire des coïncidences conduisant à l'issue tragique, n'empêche nullement le lecteur ou le spectateur qui ne croit pas au destin, de trouver une signification aux événements narrés. Pourquoi ? Parce que le récit fait "résonner» dans l'esprit du lecteur ou du spectateur une séquence émotionnelle familière : le fait de fuir un désastre d'une manière désespérée pour découvrir, avec horreur, que l'on a cessé de s'y précipiter. Le lecteur ou le spectateur, s'il ne connaît pas la raison des événements narrés, connaît cependant l'effet d'être soi-même défait de par ses propres efforts.

On peut ajouter qu'il en est des émotions ressenties à la lecture ou au spectacle des récits de fiction comme des émotions dans un contexte non fictionnel. Dans les deux cas, notre capacité à «résonner » émotionnellement ou à être «infecté » émotionnellement, pour reprendre la terminologie de Walton (1999), ne semble pas requérir que la source de notre «infection» émotionnelle

18. Je remercie M. Montminy d'avoir soulevé ce point. 
soit une personne ou soit imaginée être une personne. Nous pouvons, par exemple, être "infectés » émotionnellement par le ronronnement d'un chat, répondre émotionnellement au ronronnement du chat par un sentiment de contentement, sentiment à partir duquel nous jugeons que ce chat est lui-même dans un état de contentement. De même, nous pouvons répondre émotionnellement aux choses inanimées : l'observation de la tour de Pise - un autre exemple de Walton (1999) — peut me conduire à éprouver un état mental d'instabilité et cela, sans que je pense que la tour éprouve des sensations ou des sentiments. Nos réponses émotionnelles aux choses inanimées, comme nos réponses émotionnelles à tel ou tel passage musical plein de tension, ne requièrent pas de notre part que nous imaginions la chose inanimée ni la musique comme une personne ${ }^{19}$. De même, il ne semble pas que notre capacité à être ému par un récit de fiction requière que nous nous représentions l'auteur du récit et ses intentions narratives. C'est pourquoi la conception émotionnelle de l'interprétation des récits de fiction est, à la différence de la conception communicationnelle, une conception non intentionnaliste.

En résumé, la conception émotionnelle de l'interprétation des récits de fiction est une conception subjective de l'interprétation et, bien que ne relevant pas de la «théorie-théorie", une conception non simulationniste de l'interprétation. C'est une conception subjective de l'interprétation puisque, selon cette conception, les événements narrés sont interprétés en fonction de la manière dont ils «résonnent » émotionnellement dans l'esprit du lecteur ou du spectateur, non pour ce qu'ils sont supposés être objectivement ou selon leur auteur (inféré). C'est parce qu'un récit nous permet d'éprouver de la crainte, puis de la pitié, c'est parce qu'il nous plonge dans des états de terreur, puis de soulagement, que ce récit a un sens et une unité pour nous, aussi invraisemblables que soient les événements narrés d'un point de vue objectif. La conception émotionnelle de l'interprétation qui vient d'être esquissée n'est pas non plus une conception simulationniste de l'interprétation puisque les états émotionnels qui constituent la base de l'interprétation ne résultent pas de simulations, mais davantage de la «contagion » émotionnelle ou, plus généralement, de la « résonance» émotionnelle ${ }^{20}$.

Enfin, l'interprétation ne revient pas, selon la conception émotionnelle et à la différence de la conception communicationnelle de l'interprétation, à expliquer mais, en quelque sorte, à assimiler les événements narrés. Il s'agit pour le lecteur ou le spectateur de faire sien le déroulement des événements

19. Sur tout ceci, cf. Walton (1999).

20. Il faut noter cependant que, contrairement à ce qui vient d'être dit, Gordon soutient que la «contagion" émotionnelle est une forme primitive de simulation (Cf. Gordon, [1995, p. 113]). Si l'on souscrit à l'interprétation de Gordon, il faudrait conclure que la conception émotionnelle de l'interprétation est une conception simulationniste de l'interprétation. D'autre part, comme me l'a fait justement remarquer un éditeur de ce volume, la conception émotionnelle de l'interprétation n'exclut nullement qu'il y ait, en plus des phénomènes de "résonance » émotionnelle, des simulations des états mentaux des personnages du récit. 
afin d'en découvrir le sens. Du point de vue du contenu des événements narrés, la liberté des auteurs de récits de fiction est certes très grande. Mais cette liberté n'a de sens que dans la mesure où les récits inventés par ces auteurs « résonnent » émotionnellement dans l'esprit des lecteurs ou des spectateurs, seulement si ces récits peuvent être ressentis par ces lecteurs ou spectateurs d'une manière qui leur est familière.

\section{Conclusion}

Une histoire qui, d'un point de vue objectif, est invraisemblable peut bien souvent, après interprétation, avoir une signification pour le lecteur ou le spectateur. Selon les deux conceptions de l'interprétation des récits de fiction qui ont été ici esquissées, l'interprétation est un processus d'enrichissement sémantique, une entreprise donatrice d'intelligibilité. Alors que la conception émotionnelle de l'interprétation voit dans notre capacité à attribuer des états mentaux à autrui par "contagion» ou "résonance» émotionnelle la source de cet enrichissement sémantique, la conception communicationnelle de l'interprétation voit dans nos capacités métareprésentationnelles la source de cet enrichissement : c'est parce que le lecteur ou le spectateur se représente, d'une manière ou d'une autre, les états mentaux de l'intelligence organisatrice du récit qu'il est conduit à trouver du sens dans le récit. L'une des difficultés auxquelles se heurte la conception communicationnelle est que les enfants, dès l'âge de deux ans, semblent prendre plaisir à ces récits de fiction complexes et saisir leur signification alors même que nombreux sont ceux qui pensent, selon la conception communicationnelle, qu'ils ne possèdent pas avant trois ou quatre ans les capacités métareprésentationnelles requises pour prendre plaisir à ces récits et saisir leur sens ${ }^{21}$. Il semblerait donc qu'à cet âge précoce, les enfants auraient la capacité de comprendre les récits sans posséder le concept d'intention.

Plutôt que de trancher entre ces deux conceptions de l'interprétation, il faut sans doute reconnaître que la conception communicationnelle de l'interprétation reflète davantage la posture d'un critique distant qui chercherait à expliquer quasi-scientifiquement un récit de fiction en cherchant à élucider les intentions de son auteur (inféré), tandis que la conception émotionnelle reflète davantage la posture du lecteur ou du spectateur "immergé » dans la fiction, pour qui ni les contraintes de plausibilité ni les intentions de l'auteur (inféré) ne jouent un rôle substantiel.

Précisons ce dernier point : dans la conception émotionnelle de l'interprétation, l'intrigue est non pas expliquée de manière causale, comme c'est le cas lorsqu'il s'agit d'expliquer le comportement d'autrui de manière causale, mais elle est émotionnellement "ressentie » ou «éprouvée » par le lecteur ou le spectateur, comme c'est le cas lorsque l'on attribue une pensée à autrui sur la base des émotions que l'on ressent à son égard. En conséquence, la plausibilité 
et la rationalité ne sont plus des normes de l'interprétation dans la conception émotionnelle de l'interprétation, alors que c'était le cas dans la conception communicationnelle de l'interprétation.

Faut-il voir, dans ce dernier point, la manifestation du caractère descriptif de l'interprétation dans la conception émotionnelle, par opposition au caractère normatif de l'interprétation dans la conception communicationnelle $^{22}$ ? Certes, dans la conception communicationnelle, des normes comme la cohérence, la plausibilité et la simplicité ne cessent de guider le processus d'interprétation. Il y a donc pour la conception communicationnelle des interprétations de valeur inégale, des interprétations correctes et des interprétations incorrectes, des interprétations qui expliquent mieux que d'autres, comme on l'a vu plus haut lorsqu'une interprétation des récits de Doyle était rejetée parce qu'elle était moins explicative qu'une autre interprétation. Selon la conception émotionnelle, l'interprétation et la compréhension des récits de fiction, en tant qu'elles dépendent de nos réponses émotionnelles, ont, comme les émotions, une base biologique. Faut-il en conclure qu'aucune contrainte de type normatif ne guide l'interprétation dans la conception émotionnelle? La conception émotionnelle de l'interprétation n'exclut pas que les émotions éprouvées par le lecteur ou le spectateur aient un contenu représentationnel associé à des conditions de correction. En effet, si l'on admet que nos émotions ont un contenu représentationnel, si l'on admet par exemple que l'émotion de peur du lecteur ou du spectateur représente aux yeux de ce lecteur ou de ce spectateur tel personnage comme étant dangereux, alors l'état émotionnel du lecteur ou du spectateur est associé pour lui à des conditions de correction : ce que sa peur représente lui semble correct, et le personnage en question lui semble réellement dangereux. C'est pourquoi la conception émotionnelle de l'interprétation, bien qu'essentiellement descriptive, contient également une dimension normative, du moins si l'on admet que les émotions sont des états intentionnels représentationnels ${ }^{23}$.

Il reste que ce qui distingue les deux conceptions de l'interprétation est, avant tout, la visée assignée à l'interprétation dans chaque conception. Dans la conception communicationnelle, l'interprétation vise à produire le contenu de l'histoire au moyen d'une explication des intentions narratives de l'auteur (inféré) tandis que dans la conception émotionnelle, l'interprétation vise à produire une signification pour une histoire dont le contenu pourrait rester partiellement inexpliqué. Dans la conception communicationnelle, ce qui est visé est le contenu de l'histoire, c'est-à-dire un réseau de causes objectives dans lequel les événements narrés peuvent prendre place tandis que, dans la conception émotionnelle, ce qui est visé est la signification que chaque lecteur ou spectateur peut projeter dans l'histoire à partir de son expérience émotionnelle.

22. C'est une suggestion de M. Montminy.

23. Sur ce point, voir Döring (2003). 
Notons en terminant qu'au-delà des objections empiriques qui ont été adressées à la conception communicationnelle et qui doivent être prises au sérieux, la conception émotionnelle de l'interprétation a, sur sa rivale, l'avantage de rendre compte du processus d'interprétation à l'œuvre, semble-t-il, pour l'ensemble des récits, qu'ils soient de fiction ou non. Tout récit, qu'il relève de la fiction, de la biographie ou de la science historique, que les événements narrés aient une existence objective indépendante du récit ou non, peut donner lieu à une interprétation émotionnelle. Les auteurs de fiction, comme les auteurs de biographies ou de textes historiques, exploitent notre disposition à «résonner » émotionnellement. Il incombe alors au lecteur ou au spectateur d'y prendre garde afin de distinguer, par lui-même, les récits de fiction des autres types de récit.

\section{Références bibliographiques}

Astington, J. W., P. L. Harris et D. R. Olson, dir., Developing Theories of Mind, Cambridge, Cambridge University Press, 1988.

Banfield, A. Unspeakable sentences : Narration and Representation in the Language of Fiction, Boston, Routledge and Kegan Paul, 1987. Traduction française par C. Veken dans A. Banfield, Phrases sans paroles - Théorie du récit et du style indirect libre, Paris, Seuil, 1995. Booth, W. The Rhetoric of Fiction, Chicago, University of Chicago Press, 1961. Carruthers, P. et P. Smith, dir., Theories of Theories of Mind, Cambridge, Cambridge University Press, 1996.

Currie, G. «Interpretation and Objectivity », Mind, vol. 102, 1993, p. 413-428. . Image and Mind - Film, Philosophy and Cognitive Science, Cambridge, Cambridge University Press, 1995a. . "Unreliability refigured : Narrative in Literature and Film», The Journal of Aesthetics and Art Criticism, 53 :1, 1995b, p. 19-29. . "Narrative Desire ", in C. Plantinga and G. M. Smith, dir., Passionate Views - Film, Cognition, and Emotion, Baltimore and London, The John Hopkins University Press, 1998a, p. 183-199.

. "Tense and Egocentricity in Fiction", in R. Le Poidevin, dir., Questions of Time and Tense, Oxford, Clarendon Press, 1998b, p. 265-283.

Currie, G et J. Jureidini. "Art and Delusion» The Monist, An International Quaterly Journal of General Philosophical Inquiry, 2003.

Davies, M. et T. Stone, dir., Folk Psychology: The Theory of Mind Debate, Oxford, Blackwell, 1995a.

. dir., Mental Simulation : Evaluations and Applications, Oxford, Blackwell, 1995b.

DeLancey, C. Passionate Engines : What Emotions Reveal about Mind and Articifical Intelligence, Oxford, Oxford University Press, 2002.

Dokic, J et J. Proust, dir., Simulation and Knowledge of Action, John Benjamins Publishing Company, Advances in Consciousness Research series, Amsterdam/Philadelphia, 2002. 
Döring, S. A. «Explaining Action by Emotion », The Philosophical Quaterly, vol. 53, n 211, 2003, p. 214-230.

Duchan, J., G. Bruder et L. Hewitt, dir., Deixis in Narrative - A Cognitive Science Perspective, Hillsdale, New Jersey, Lawrence Erlbaum Associates, 1995.

Glenberg, A. M., M. Meyer, et K. Lindem. «Mental Models Contribute to Foregrounding during Text Comprehension ", Journal of Memory and Language, 26, 1987, p. 69-83.

Gordon, R. "The Simulation Theory : Objections and Misconceptions ", in Davies et Stone, dir., 1995, p. 100-122.

Hamburger, K. The Logic of Literature [Die Logik der Dichtung 1957 Stuttgart, Ernst Klett Verlag] M. J. Rose, 1973. Trad. Bloomington, Indiana University Press. Traduction française de P. Cadiot, Logique des genres littéraires, Paris, Éditions du Seuil, 1986.

Kuroda, S-Y. «Reflections on the foundations of narrative theory - from a linguistic point of view ", in T. A. van Dijk, dir., Pragmatics of Language and Literature, Amsterdam, North-Holland, 1976, p. 107-140.

Morrow, D. G., S. L Greenspan et G. H. Bower, «Accessibility and Situation Models in Narrative Comprehension ", Journal of Memory and Language, 26, 1987, p. 165-187.

Morrow, D. G., G. H. Bower, et S. L. Greenspan «Updating Situation Models during Narrative Comprehension ", Journal of Memory and Language, 28, 1989, p. 292-312.

Perner, J. Understanding the Representational Mind, Cambridge (Mass.), Bradford/MIT Press, 1991.

Rall, J. et P. L. Harris. «In Cinderella's Slippers? Story Comprehension from the Protagonist's Point of View ", Developmental Psychology, 36, 2000, p. 202-208.

Schank, R. C. et R. P. Abelson. Scripts, plans, goals and understanding, Hillsdale (NJ), Lawrence Erlbaum Associates, 1977.

Segal, E. M. "A Cognitive-Phenomenological Theory of Fictional Narrative", in J. Duchan, G. Bruder et L. Hewitt, dir., Deixis in Narrative - A Cognitive Science Perspective, Hillsdale (NJ), Lawrence Erlbaum Associates, Inc., 1995, p. 61-78.

Velleman, D. «Narrative Explanation », 2002, ms non publié.

Walton, K. «Projectivism, Empathy, and Musical Tension», Philosophical Topics, vol. 26, nos 1 et 2, 1999, p. 407-440.

Wellman, H. M. The Child's Theory of Mind, Cambridge (Mass.), Bradford/MIT Press, 1990.

Zwaan, R. A., C. J. Madden et S. N Whitten, "The Presence of an Event in the Narrated Situation Affects its Availability to the Comprehender", Memory and Cognition, 28 (6), 2000, p. 1022-1028. 